\title{
A HAUSDORFF MEASURE INEQUALITY
}

\author{
BY
}

\author{
LAWRENCE R. ERNST $\left({ }^{1}\right)$ AND GERALD FREILICH
}

\begin{abstract}
We prove that the Hausdorff $(m+k)$-measure of a product set is no less than the product of the Hausdorff $m$-measure of the (measurable) first component set in $\mathrm{R}^{m}$ and the (finite) Hausdorff $k$-measure of the second component in $\mathrm{R}^{\boldsymbol{n}}$.
\end{abstract}

1. Introduction. We study the relationship between the Hausdorff measure of product sets and the Hausdorff measures of their components. It is proven in $[3,2.10 .45]$ that if $V \subset \mathbf{R}^{n}$ with $H^{k}(V)<\infty$, then there exists a real number $c$ such that $H^{m+k}(U \times V)=c H^{m}(U) H^{k}(V)$ for every $H^{m}$-measurable subset $U$ of $\mathbf{R}^{m}$, where $H^{t}$ denotes Hausdorff $t$-dimensional measure. (Note that in $\mathbf{R}^{m}, H^{m}$ reduces to Lebesgue $m$-dimensional measure, $L^{m}$.) It is known that $c=1$ if $V$ is $k$-rectifiable $[3,3.2 .23]$, and that $c>1$ for some $V$ [1], [4].

In $[3,2.10 .46]$ the question was posed whether there exists $V$ for which $c<1$. In this paper we present a negative answer to that question. This general result, Theorem 3.6, follows easily from Theorem 3.5 which is the special case when $m=1, V$ is a Borel set and $U=[0,1]$. The proof of Theorem 3.5 depends on our method of obtaining the measure of the product set; specifically we apply a Hausdorff gauge construction using coverings consisting of sets having a useful symmetry property (Theorem 3.4 ).

The authors wish to thank the referee for several suggestions, particularly the improvement of Lemma 3.2.

2. Preliminaries. In general we adopt the notation and terminology of [3]. Presented in this section is additional notation that we use.

Throughout this paper $k$ is a nonnegative real number and $r=k+1, A$ is a Borel subset of $\mathrm{R}^{n}$ with $H^{k}(A)<\infty, I=\{x: 0 \leqslant x \leqslant 1\}$ and $E=I \times A$.

Let $p: \mathbf{R} \times \mathbf{R}^{n} \rightarrow \mathbf{R}, q: \mathbf{R} \times \mathbf{R}^{n} \rightarrow \mathbf{R}^{n}, p(x, y)=x, q(x, y)=y$ for $x \in$ $\mathbf{R}, y \in \mathbf{R}^{n}$.

For $S$ a subset of some metric space, $t$ a nonnegative real number let

$$
h^{t}(S)=\alpha(t) 2^{-t}(\operatorname{diam} S)^{t}=\left[\Gamma(1 / 2)^{t} / \Gamma(t / 2+1)\right] 2^{-t}(\operatorname{diam} S)^{t}
$$

Received by the editors July 26, 1974 and, in revised form, April 1, 1975.

AMS (MOS) subject classifications (1970). Primary 28A35, $28 \mathrm{~A} 75$.

Key words and phrases. Hausdorff measure, product set.

(1) Research supported by a grant from the City University of New York Faculty Research Award Program. 
if $S \neq \varnothing$, and $h^{t}(\varnothing)=0$. This is the gauge function used in defining $H^{t}$ $[3,2.10 .2]$. Also let $\lambda(S, t)$ denote the family of all countable coverings of $S$ consisting of nonempty sets of diameter not exceeding $t$.

For $S \subset \mathbf{R} \times \mathbf{R}^{n}, a \in \mathbf{R} \times \mathbf{R}^{n}$ define $\xi(S, a)=S \cap q^{-1}\{q(a)\}$.

Finally let $\Omega$ denote the family of all nonempty subsets $D$ of $E$ such that diam $D<\infty, D$ is closed in $E$, and $D$ is symmetric with respect to

$$
p^{-1}\{[\inf p(D)+\sup p(D)] / 2\} \text {. }
$$

3. Principal results.

3.1. Lemma. If $D$ is $H^{r}$-measurable, $D \subset C \subset E, \infty>\epsilon>0, \delta>0$, and $\Sigma_{S \in F} h^{r}(S) \geqslant H^{r}(C)-\epsilon$ for all $F \in \lambda(C, \delta)$, then $\Sigma_{S \in W} h^{r}(S) \geqslant H^{r}(D)-\epsilon$ for all $W \in \lambda(D, \delta)$.

Proof. For any $\epsilon_{1}>0$, choose $K \in \lambda(C \sim D, \delta)$ satisfying $\Sigma_{S \in K} h^{r}(S) \leqslant$ $H^{r}(C \sim D)+\epsilon_{1}$. Then $W \cup K \in \lambda(C, \delta)$ for all $W \in \lambda(D, \delta)$. Consequently

$$
\sum_{S \in W} h^{r}(S) \geqslant \sum_{S \in W \cup K} h^{r}(S)-\sum_{S \in K} h^{r}(S) \geqslant H^{r}(D)-\epsilon-\epsilon_{1} .
$$

3.2. LEMMA. If $S$ is a bounded $H^{r}$-measurable subset of $\mathbf{R} \times A, \lambda \in \mathbf{R}$ and $\sigma(S)$ is the Steiner symmetrization of $S$ with respect to $p^{-1}(\lambda)[3,2.10 .30]$, then $\sigma(S)$ is $H^{r}$-measurable and $H^{r}(S)=H^{r}[\sigma(S)]$.

Proof. Let

$\Phi=\left\{S \subset \mathbf{R} \times \mathbf{R}^{n}: S\right.$ is bounded, $(\mathbf{R} \times A) \cap S$ and $\sigma[(\mathbf{R} \times A) \cap S]$

are $H^{r}$-measurable, $\left.H^{r}[(\mathbf{R} \times A) \cap S]=H^{r}(\sigma[(R \times A) \cap S])\right\}$.

The proof consists of four parts.

Part 1. If $B_{i}$ is an open ball in $\mathrm{R} \times \mathrm{R}^{n}$ for $i=1, \ldots, j$, then $\bigcup_{i=1}^{j} B_{i} \in \Phi$.

Proof. For each $B_{i}$, the functions $\sup p\left(\xi\left[B_{i},(\lambda, x)\right]\right)$ and inf $p\left(\xi\left[B_{i},(\lambda, x)\right]\right)$ are uniformly continuous for $x \in q\left(B_{i}\right)$. It follows that if $\epsilon>0$ and $B=(\mathrm{R} \times A) \cap \bigcup_{i=1}^{j} B_{i}$, there is a $\delta>0$ for which $D \subset A$ and diam $D<\delta$ implies

$$
H^{1}\left[\bigcup_{x \in D} p(\xi[B,(\lambda, x)]) \sim \bigcap_{x \in D} p(\xi[B,(\lambda, x)])\right]<\epsilon .
$$

If we take a Borel partition $F$ of $A$ such that diam $D<\delta$ for all $D \in F$, then $[3,2.10 .45]$ implies

$$
\left|H^{r}(B)-H^{r}[\alpha(B)]\right|<\epsilon \cdot \alpha(r)[\alpha(k)]^{-1} 2^{(r-2) / 2} H^{r}(A) .
$$

To conclude the proof we let $\epsilon$ approach zero.

Part 2. If $S_{i} \in \Phi, S_{i} \subset S_{i+1}$ for $i=1,2, \ldots$ and the $S_{i}$ are uniformly bounded, then $\bigcup_{i=1}^{\infty} S_{i} \in \Phi$. 
Proof. For $\rho>1$, let $f_{\rho}$ be the Lipschitzian mapping $(x+\lambda, y) \in \mathbf{R} \times$ $\mathbf{R}^{n} \rightarrow(\rho x+\lambda, y)$. Then $H^{r}\left[f_{\rho}(X)\right] \leqslant \rho^{r} H^{r}(X)$ for all $X \subset \mathbf{R} \times \mathbf{R}^{n}$. Since

$$
\bigcup_{i=1}^{\infty} \sigma\left[(\mathrm{R} \times A) \cap S_{i}\right] \subset \sigma\left[(\mathrm{R} \times A) \cap \bigcup_{i=1}^{\infty} S_{i}\right] \subset f_{\rho}\left(\bigcup_{i=1}^{\infty} \sigma\left[(\mathrm{R} \times A) \cap S_{i}\right]\right)
$$

for all $\rho>1$, we conclude that

$$
H^{r}\left(\bigcup_{i=1}^{\infty} \sigma\left[(\mathrm{R} \times A) \cap S_{i}\right]\right)=H^{r}\left(\sigma\left[(\mathrm{R} \times A) \cap \bigcup_{i=1}^{\infty} S_{i}\right]\right) .
$$

Furthermore, since $\bigcup_{i=1}^{\infty} \sigma\left[(\mathrm{R} \times A) \cap S_{i}\right]$ is $H^{r}$-measurable, so is $\sigma\left[(\mathrm{R} \times A) \cap \bigcup_{i=1}^{\infty} S_{i}\right]$. Finally

$$
\begin{array}{r}
H^{r}\left[(\mathrm{R} \times A) \cap \bigcup_{i=1}^{\infty} S_{i}\right]=\lim _{i \rightarrow \infty} H^{r}\left[(\mathrm{R} \times A) \cap S_{i}\right]=\lim _{i \rightarrow \infty} H^{r}\left(\sigma\left[(\mathrm{R} \times A) \cap S_{i}\right]\right) \\
=H^{r}\left(\bigcup_{i=1}^{\infty} \sigma\left[(\mathrm{R} \times A) \cap S_{i}\right]\right)=H^{r}\left(\sigma\left[(\mathrm{R} \times A) \cap \bigcup_{i=1}^{\infty} S_{i}\right]\right) .
\end{array}
$$

Part 3. If $S_{i} \in \Phi, S_{i}$ is a Borel set, $S_{i} \supset S_{i+1}$ for $i=1,2, \ldots$, then $\bigcap_{i=1}^{\infty} S_{i} \in \Phi$.

PROOF. Since

$$
\begin{aligned}
\bigcap_{i=1}^{\infty} \sigma[(\mathrm{R} \times A) & \left.\cap S_{i}\right] \\
= & \sigma\left[(\mathrm{R} \times A) \cap \bigcap_{i=1}^{\infty} S_{i}\right] \cup\left[\{\lambda\} \times q\left(\bigcap_{i=1}^{\infty} \sigma\left[(\mathrm{R} \times A) \cap S_{i}\right]\right)\right]
\end{aligned}
$$

we see that $\sigma\left[(R \times A) \cap \bigcap_{i=1}^{\infty} S_{i}\right]$ is $H^{r}$-measurable and that

$$
\begin{aligned}
& H^{r}\left[(\mathrm{R} \times A) \cap \bigcap_{i=1}^{\infty} S_{i}\right]=\lim _{i \rightarrow \infty} H^{r}\left[(\mathrm{R} \times A) \cap S_{i}\right] \\
& \quad=\lim _{i \rightarrow \infty} H^{r}\left(\sigma\left[(\mathrm{R} \times A) \cap S_{i}\right]\right)=H^{r}\left(\sigma\left[(\mathrm{R} \times A) \cap \bigcap_{i=1}^{\infty} S_{i}\right]\right) .
\end{aligned}
$$

Part 4. If $S$ is a bounded $H^{r}$-measurable subset of $R \times A$, then $S \in \Phi$.

Proof. By Parts 1, 2 and 3, we see that bounded open, $G_{\delta}$ - and $G_{\delta \sigma}$-sets are in $\Phi$. Now we may choose bounded $G_{\delta \sigma}$-sets $S_{1}$ and $S_{2}$ so that $S_{2} \subset S \subset$ $S_{1}$ and $H^{r}\left(S_{2}\right)=H^{r}(S)=H^{r}\left(S_{1}\right)$. Then $\sigma\left(S_{2}\right) \subset \sigma(S) \subset \sigma\left[(R \times A) \cap S_{1}\right]$ and therefore

$$
\begin{aligned}
H^{r}\left(S_{2}\right) & =H^{r}\left[\sigma\left(S_{2}\right)\right] \leqslant H^{r}[\sigma(S)] \leqslant H^{r}\left(\sigma\left[(\mathbf{R} \times A) \cap S_{1}\right]\right) \\
& =H^{r}\left[(\mathbf{R} \times A) \cap S_{1}\right]=H^{r}\left(S_{2}\right) .
\end{aligned}
$$


It follows that $H^{r}(S)=H^{r}[\sigma(S)]$ and that $\sigma(S)$ is $H^{r}$-measurable.

REMARK. It is easy to extend Lemma 3.2 to unbounded sets.

3.3. Lemma. If $\eta>0, \delta>0, B \subset E, H^{r}(B)>0$ and $E \sim B=\bigcup Q$ for some finite subset $Q$ of $\Omega$, then there exists $G \in \lambda(\bigcup G, \delta)$ such that $\bigcup G \subset B$, $G$ is a finite subset of $\Omega$, and

(i) $H^{r}(\bigcup G) \geqslant 10^{-2 r} H^{r}(B)$,

(ii) $\Sigma_{S \in G} h^{r}(S) \leqslant H^{r}(\bigcup G)+\eta$.

Proof. Choose $\delta_{1}>0$ so that if $M=B \cap\left\{x: \operatorname{dist}(x, E \sim B)>\delta_{1}\right\}$, then

$$
H^{r}(M) \geqslant H^{r}(B) / 2 .
$$

Let $\epsilon=\inf \left\{10^{-2} r H^{r}(M), \eta / 2\right\}$, choose $\delta_{2}>0$ satisfying

$$
\sum_{S \in F} h^{r}(S) \geqslant H^{r}(M)-\epsilon \text { for all } F \in \lambda\left(M, \delta_{2}\right),
$$

and let $\gamma=\inf \left\{\delta_{,} \delta_{1}, \delta_{2} / 12\right\}$. Then choose $K \in \lambda(M, \gamma)$ consisting of sets closed in $M$ such that

$$
\sum_{S \in K} h^{r}(S) \leqslant H^{r}(M)+\epsilon .
$$

Let $K_{1}$ be a finite subset of $K$ such that

$$
H^{r}\left(U K_{1}\right) \geqslant H^{r}(M)-\epsilon .
$$

For $S \in K_{1}$ denote the Steiner symmetrization of $S$ with respect to $p^{-1}([\inf p(S)+\sup p(S)] / 2)$ by $\mu(S)$, and then let $K_{2}=\left\{\mu(S): S \in K_{1}\right\}$. For each $T \in K_{2}$ choose $\zeta(T) \in K_{1}$ satisfying the conditions $\mu[\zeta(T)]=T$ and

$$
\operatorname{diam} \zeta(T)=\sup \left\{\operatorname{diam} S: S \in K_{1} \text { and } \mu(S)=T\right. \text { \}. }
$$

We then apply $[3,2.8 .4]$ to obtain a disjointed subfamily $G$ of $K_{2}$ such that

$$
K_{2}=\bigcup_{S \in G}\left\{T: T \in K_{2}, T \cap S \neq \varnothing, \operatorname{diam} \zeta(T) \leqslant 2 \operatorname{diam} \zeta(S)\right\} .
$$

Clearly now $G \in \lambda(\cup G, \delta)$ since

$$
\operatorname{diam} \mu(S) \leqslant \operatorname{diam} S \text { for all } S \in K_{1} ;
$$

$\bigcup_{G} \subset B$ since

$$
\operatorname{diam} \xi[S \cup \mu(S), x] \leqslant \operatorname{diam} S \text { for all } S \in K_{1}, x \in S \cup \mu(S) ;
$$

and $G$ is a finite subset of $\Omega$.

To establish (i) and (ii) and thus complete the proof of the lemma we first let $Z=\{\zeta(T): T \in G\}$, and choose for each $S \in Z$ a closed ball, denoted by $\psi(S)$ such that the center of $\psi(S)$ is in $S$ and 


$$
\operatorname{diam} \psi(S)=12 \operatorname{diam} S .
$$

From (7) and the fact that $q[\mu(S)]=q(S)$ for all $S \in K_{1}$ we obtain that $\operatorname{diam}[S \cup \mu(S)] \leqslant 2 \operatorname{diam} S$ for all $S \in K_{1}$, which we combine with (5) and (8) to deduce that $\bigcup_{K_{1}} \subset U_{S \in Z} \psi(S)$; consequently

$$
\{\psi(S): S \in Z\} \in \lambda\left(\cup K_{1}, \delta_{2}\right) .
$$

From (8), Lemma 3.1, (2), (9) and (4) we then obtain

$$
\begin{aligned}
\sum_{S \in Z} h^{r}(S) & =12^{-r} \sum_{S \in Z} h^{r}[\psi(S)] \geqslant 12^{-r}\left[H^{r}\left(U K_{1}\right)-\epsilon\right] \\
& \geqslant 12^{-r}\left[H^{r}(M)-2 \epsilon\right] \geqslant 12^{-r} H^{r}(M)-\epsilon .
\end{aligned}
$$

We next use (3), Lemma 3.1 and (2) to find

$$
\begin{aligned}
\sum_{S \in Z} h^{r}(S) & \leqslant H^{r}(M)+\epsilon-\sum_{S \in K \sim Z} h^{r}(S) \\
& \leqslant H^{r}(M)+2 \epsilon-H^{r}(M \sim \cup Z)=H^{r}(\bigcup Z)+2 \epsilon .
\end{aligned}
$$

From (6), (11), Lemma 3.2 and the fact that $G$ is disjointed we then deduce

$$
\begin{aligned}
\sum_{S \in G} h^{r}(S) & \leqslant \sum_{S \in Z} h^{r}(S) \leqslant H^{r}(\bigcup Z)+2 \epsilon \\
& \leqslant \sum_{S \in Z} H^{r}(S)+2 \epsilon=\sum_{S \in G} H^{r}(S)+2 \epsilon=H^{r}(\bigcup G)+2 \epsilon,
\end{aligned}
$$

which yields (ii). Furthermore (i) now follows from (12), (10) and (1) since

$$
\begin{aligned}
H^{r}\left(\bigcup_{G}\right) & \geqslant \sum_{S \in Z} h^{r}(S)-2 \epsilon \geqslant 12^{-r} H^{r}(M)-3 \epsilon \\
& \geqslant 2 \cdot 10^{-2 r} H^{r}(M) \geqslant 10^{-2 r} H^{r}(B) .
\end{aligned}
$$

3.4. THEOREM. If $\epsilon>0, \delta>0$, then there exists $F \in \lambda(E, \delta)$ such that $F \subset \Omega$ and $\Sigma_{s \in F} h^{r}(S) \leqslant H^{r}(E)+\epsilon$.

Proof. We construct a sequence $G_{0}, G_{1}, G_{2}, \ldots$ inductively as follows: we let $G_{0}=\varnothing$ and obtain $G_{m}$ from $G_{0}, \ldots, G_{m-1}$ by letting $G_{m}=\varnothing$ if $H^{r}\left(E \sim \bigcup_{i=0}^{m-1} \cup G_{i}\right)=0$ and otherwise applying Lemma 3.3 with $B=E \sim$ $\bigcup_{i=0}^{m-1} \cup G_{i}, \eta=2^{-m-1} \epsilon$ to obtain $G_{m} \in \lambda\left(\bigcup G_{m}, \delta\right)$ satisfying $\bigcup G_{m} \subset E \sim$ $\bigcup_{i=0}^{m-1} \cup G_{i}, G_{m}$ is a finite subset of $\Omega$,

$$
H^{r}\left(\cup G_{m}\right) \geqslant 10^{-2 r} H^{r}\left(E \sim \bigcup_{i=0}^{m-1} \cup G_{i}\right),
$$




$$
\sum_{S \in G_{m}} h^{r}(S) \leqslant H^{r}\left(U G_{m}\right)+2^{-m-1} \epsilon .
$$

From (13) we deduce that

$$
\sum_{m=1}^{j} H^{r}\left(\bigcup G_{m}\right) \geqslant\left[1-\left(1-10^{-2} \eta^{j}\right] H^{r}(E)\right.
$$

for all positive integers $j$; consequently

$$
H^{r}\left(E \sim \bigcup_{m=1}^{\infty} \cup G_{m}\right)=H^{r}(E)-\sum_{m=1}^{\infty} H^{r}\left(U G_{m}\right) \leqslant H^{r}(E)-H^{r}(E)=0,
$$

which together with $[3,2.10 .42]$ establishes that there exists $K \in$ $\lambda\left(E \sim \bigcup_{m=1}^{\infty} \cup G_{m}, \delta\right)$ consisting of closed balls such that

$$
\sum_{S \in K} h^{r}(S) \leqslant \epsilon / 2
$$

Finally we let $F=\bigcup_{m=1}^{\infty} G_{m} \cup K$; note that clearly $F \in \lambda(E, \delta)$ and $F \subset \Omega$, and combine (14), (15) to obtain $\Sigma_{S \in F} h^{r}(S) \leqslant H^{r}(E)+\epsilon$.

3.5. THEOREM. $H^{k+1}(E) \geqslant H^{k}(A)$.

Proof. Consider any $\epsilon>0$. Let $\delta>0$ be such that $\Sigma_{S \in G^{h}} h^{k}(A) \geqslant H^{k}(A)-\epsilon$ for every $G \in \lambda(A, \delta)$. By Theorem 3.4 there exists $F \in \lambda(E, \delta)$ satisfying $F \subset$ $\Omega$ and $\Sigma_{S \in F} h^{k+1}(S) \leqslant H^{k+1}(E)+\epsilon$. We observe that to complete the proof it suffices to show that

$$
\int_{0}^{1} h^{k}\left[p^{-1}(x) \cap S\right] d L^{1} x \leqslant h^{k+1}(S) \text { for all } S \in F,
$$

since we would then have

$$
\begin{aligned}
H^{k}(A)-\epsilon & \leqslant \inf \left\{\sum_{S \in F} h^{k}\left[p^{-1}(x) \cap S\right]: x \in I\right\} \\
& \leqslant \int_{0}^{1} \sum_{S \in F} h^{k}\left[p^{-1}(x) \cap S\right] d L^{1} x \leqslant \sum_{S \in F} h^{k+1}(S) \leqslant H^{k+1}(E)+\epsilon .
\end{aligned}
$$

To prove (16) we consider any $S \in F$, let $\gamma=\operatorname{diam} S$, and $c=$ [inf $p(S)+\sup p(S)] / 2$. Since $S \in \Omega$, it is symmetric with respect to $p^{-1}(c)$ and so if $(x, y) \in S$ then $(2 c-x, y) \in S$ also. It follows that

$$
\operatorname{diam}\left[p^{-1}(x) \cap S\right] \leqslant\left[\gamma^{2}-4(x-c)^{2}\right]^{1 / 2} \text { for all } x \in[c-\gamma / 2, c+\gamma / 2] .
$$

We then use this inequality, together with the substitutions $u=2(x-c) / \gamma$ and $t=u^{2}$, the relation

$$
\Gamma(1 / 2) \Gamma[(k+2) / 2] / \Gamma[(k+3) / 2]=\int_{0}^{1} t^{-1 / 2}(1-t)^{k / 2} d L^{1} t
$$

between the beta and gamma functions, and the definition of $\alpha$ to compute 


$$
\begin{aligned}
\int_{0}^{1} h^{k}\left[p^{-1}(x)\right. & \cap S] d L^{1} x \leqslant \alpha(k) 2^{-k} \int_{c-\gamma / 2}^{c+\gamma / 2}\left[\gamma^{2}-4(x-c)^{2}\right]^{k / 2} d L^{1} x \\
& =\alpha(k) 2^{-k} \gamma^{k+1} \int_{0}^{1}\left(1-u^{2}\right)^{k / 2} d L^{1} u \\
& =\alpha(k) 2^{-(k+1)} \gamma^{k+1} \int_{0}^{1} t^{-1 / 2}(1-t)^{k / 2} d L^{1} t \\
& =\alpha(k) 2^{-(k+1)} \gamma^{k+1} \Gamma(1 / 2) \Gamma[(k+2) / 2] / \Gamma[(k+3) / 2] \\
& =\alpha(k+1) 2^{-(k+1)} \gamma^{k+1}=h^{k+1}(S) .
\end{aligned}
$$

3.6. THEOREM. If $U$ is a $L^{m}$-measurable subset of $\mathbf{R}^{m}, V \subset \mathbf{R}^{n}$ and $H^{k}(V)<\infty$, then $H^{m+k}(U \times V) \geqslant L^{m}(U) H^{k}(V)$.

Proof. We first prove the special case when $m=1$ and $U=I$. Using the Borel regularity of Hausdorff measure we choose Borel sets $X \subset \mathbf{R}^{n}, Y \subset \mathbf{R} \times$ $\mathbf{R}^{n}$ so that $V \subset X, H^{k}(V)=H^{k}(X), I \times V \subset Y, H^{k+1}(I \times V)=H^{k+1}(Y)$. Let $Z=(I \times X) \sim Y$. Then $Z$ is a Borel set and therefore $q(Z)$ is $H^{k}$-measurable. Also $q(Z) \subset X \sim V$ so that $H^{k}(X)=H^{k}(V)$ implies $H^{k}[q(Z)]=0$. This in turn implies that $H^{k+1}[I \times q(Z)]=0$. Since $Z \subset I \times q(Z)$, we conclude that $H^{k+1}(Z)$ $=0$ and therefore

$$
H^{k+1}(I \times V)=H^{k+1}(Y) \geqslant H^{k+1}(I \times X)
$$

From Theorem 3.5 we infer that

$$
H^{k+1}(I \times X) \geqslant H^{k}(X)=L^{1}(I) H^{k}(V)
$$

so that $H^{k+1}(I \times V) \geqslant L^{1}(I) H^{k}(V)$.

Using induction on $m$ we deduce that the conclusion holds for all positive integers $m$ if $U=I^{m}$. This in turn implies that the conclusion of the theorem is true for an arbitrary $L^{m}$-measurable subset $U$ of $\mathbf{R}^{m}[3,2.10 .45]$.

3.7. REMARK. If $s, t$ are integers, $2<t<s$, then Theorem 3.6 provides a simple example of a set $B$ in $\mathbf{R}^{s}$ for which $0<C^{t}(B)<H^{t}(B)<\infty$, where $C^{t}$ is Carathéodory $t$-dimensional measure $[3,2.10 .4]$. To construct $B$ first let $V \subset$ $\mathrm{R}^{3}$ be such that $0<C^{2}(V)<H^{2}(V)<\infty[1]$, [4], [5]. If $U=I^{t-2}$, then Theorem 3.6 implies

$$
H^{t}(U \times V) \geqslant L^{t-2}(U) H^{2}(V)=H^{2}(V),
$$

while by $[3,2.10 .46]$ we have

$$
C^{t}(U \times V) \leqslant L^{t-2}(U) C^{2}(V)=C^{2}(V) .
$$

Hence $0<C^{t}(U \times V)<H^{t}(U \times V)<\infty . B$ is then obtained by isometrically embedding $U \times V$ in $\mathbf{R}^{s}$. 


\section{BIBLIOGRAPHY}

1. A. S. Besicovitch and P. A. P. Moran, The measure of product and cylinder sets, J. London Math. Soc. 20 (1945), 110-120. MR 8, 18.

2. L. R. Ernst, $A$ proof that $\mathrm{H}^{2}$ and $\mathrm{T}^{2}$ are distinct measures, Trans. Amer. Math. Soc. 191 (1974), 363-372.

3. H. Federer, Geometric measure theory, Die Grundlehren der math. Wissenschaften, Band 153, Springer-Verlag, Berlin and New York, 1969. MR 41 \#1976.

4. G. Freilich, On the measure of Cartesian product sets, Trans. Amer. Math. Soc. 69 (1950), 232-275. MR 12, 324.

5. E. F. Moore, Convexly generated k-dimensional measures, Proc. Amer. Math. Soc. 2 (1951), 597-606. MR 13, 218.

DEPARTMENT OF MATHEMATICS, QUEENS COLLEGE OF THE CITY UNIVERSITY OF NEW YORK, FLUSHING, NEW YORK 11367 